\section{Atmung \\ Pneumothorax: Notfalldiagnostik mit dem Ultraschall}

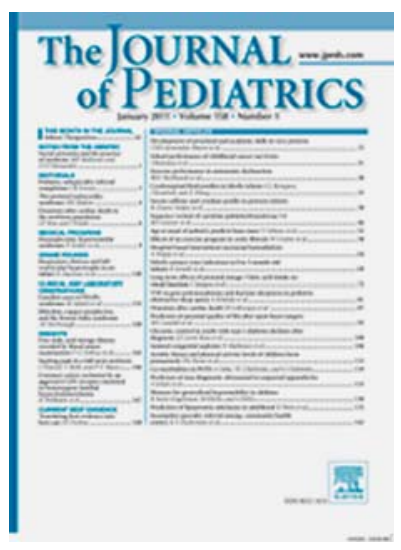

Raimondi F et al. Lung Ultrasound for Diagnosing Pneumothorax in the Critically III Neonate. J Pediatr 2016; 175: $74-78$

Etwa jedes zehnte Frühgeborene erleidet einen Pneumothorax und dekompensiert plötzlich mit einer stark gesteigerten Mortalität. Eine rasche und zuverlässige Diagnostik ohne Strahlenbelastung ist wünschenswert. Bei erwachsenen Patienten war der Ultraschall dem konventionellen Röntgen überlegen. Die Studie belegte nun, dass die Methode auch bei Frühgeborenen effektiv ist.

An der prospektiven Multicenterstudie nahmen Kliniken in Frankreich und Südeuropa teil. Eine plötzliche Dekompensation war definiert als rasche allgemeine Verschlechterung mit Abnahme der Sauerstoffsättigung $<65 \%$ und Bradykardie, Beatmungsnotwendigkeit, kardiopulmonale Reanimation und eine plötzliche Zunahme des Sauerstoffbedarfs um $\geq 50 \%$. Ausschlusskriterien waren u.a. Zwerchfellhernien und zystische Malformationen. Speziell trainierte Ärzte führten den Ultraschall mit einem hochauflösenden Schallkopf $(10 \mathrm{MHz})$ durch. Referenzstandard war eine Röntgenaufnahme des Thorax (Rückenlage, anterior-posteriore Projektion). Diagnosekriterien für den Ultraschall waren
- das Fehlen von Lungengleiten,

- die Darstellung des Lungenpunktes,

- ausbleibende Kometenschweifartefakte,

- ein Stratosphäre-Zeichen.

Insgesamt 42 Frühgeborene erfüllten die Einschlusskriterien (1531g, Gestationsalter 31 Wochen median). $27 \%$ erhielten eine nicht invasive Atemunterstützung und $58 \%$ eine Maschinenbeatmung. $53 \%$ hatten vor der Akutdekompensation Surfactant bekommen. In 26 Fällen (61,9\%) bestätigte sich ein Pneumothorax als Ursache der plötzlichen kardiorespiratorischen Verschlechterung.

Die klinische Diagnostik hatte eine Sensitivität von $84 \%$, Spezifität von $56 \%$, einen positiven prädiktiven Wert von $76 \%$ und einen negativen prädiktiven Wert von 69\%. Der Ultraschall erzielte bei allen Parametern $100 \%$. Somit konnte ein Pneumothorax nicht nur sicher diagnostiziert, sondern auch zuverlässig ausgeschlossen werden. Maßgeblich waren ein fehlendes Lungengleiten und die Bestimmung des Lungenpunktes, an dem sich die viszerale von der paritealen Pleura abhebt (Sensitivität und Spezifität jeweils 100\%). Die Untersuchung des Stratosphäre-Zeichens erfolgte lediglich bei 32 Patienten.

26 Kinder erhielten eine Thorax-Drainage. Komplikationen, z. B. Hypothermien, kamen nicht vor. Kein Säugling starb.

FAZIT

Bei den besonders gefährdeten Frühgeborenen eignete sich der Ultraschall für die zuverlässige Schnelluntersuchung eines Pneumothorax: In durchschnittlich 5,3 Minuten stand die Diagnose fest (Röntgen 19 Minuten) und ermöglichte rasche Folgemaßnahmen. Die Autoren empfehlen das Erlernen der Methode und verweisen auf den Vorteil der ausbleibenden Strahlenbelastung in dieser hochsensiblen Population.

Dr. Susanne Krome, Melle 